\title{
Ex-Vivo Gene Therapy Using Lentiviral Mediated Gene Transfer Into Umbilical Cord Blood Derived Stem Cells
}

\author{
Hanieh Jalali, ${ }^{1,}$ Kazem Parivar, ${ }^{2}$ Masoud Soleimani, ${ }^{3}$ Mohammad Nabiuni, ${ }^{4}$ and Hamid \\ Aghaee-Bakhtiari ${ }^{5}$ \\ ${ }^{1}$ Department of Developmental Biology, Faculty of Biological Sciences, Kharazmi University, Tehran, IR Iran \\ ${ }^{2}$ Department of Biology, Sciences and Research Branch, Islamic Azad University, Tehran, IR Iran \\ ${ }^{3}$ Department of Hematology, Faculty of Medical Sciences, Tarbiat Modares University, Tehran, IR Iran \\ ${ }^{4}$ Department of Cell and Molecular Biology, Faculty of Biological Sciences, Kharazmi University, Tehran, IR Iran \\ ${ }^{5}$ Department of Molecular Biology and Genetic Engineering, Stem Cells Technology Research Center, Tehran, IR Iran \\ "Corresponding author: Hanieh Jalali, Department of Developmental Biology, Faculty of Biological Sciences, Kharazmi University, Tehran, IR Iran. E-mail: std_jalali@khu.ac.ir
}

Received 2014 September 17; Accepted 2014 November 22.

\begin{abstract}
Background: Introduction of therapeutic genes into the injured site of nervous system can be achieved using transplantation of cellular vehicles containing desired gene. To transfer exogenous genes into the cellular vehicles, lentiviral vectors are one of interested vectors because of advantages such high transduction efficiency of dividing and non-dividing cells. Unrestricted somatic stem cells are subclasses of umbilical cord blood derived stem cells which are appreciate candidates to use as cellular vehicles for ex vivo gene therapy of nervous system.

Objectives: In current study we investigated the effect of lentiviral vector transduction on the neuronal related features of unrestricted somatic stem cells to indicate the probable and unwanted changes related to transduction procedure.

Materials and Methods: In this experimental study, lentiviral vector containing green fluorescent protein (GFP) were transduced into unrestricted somatic stem cells and its effect was investigated with using MTT assay, qPCR and immunohistochemistry techniques. For statistical comparison of real time PCR results, REST software (2009, Qiagen) was used.

Results: Obtained results showed lentiviral vector transduction did not have cytotoxic effects on unrestricted somatic stem cells and did not change neuronal differentiation capacity of them as well the expression of some neuronal related genes and preserved them in multilineage situation.

Conclusions: In conclusion, we suggested that lentiviral vectors could be proper vectors to transfer therapeutic gene into unrestricted somatic stem cells to provide a cellular vehicle for ex vivo gene therapy of nervous system disorders.
\end{abstract}

Keywords: Ex Vivo, Gene Therapy, Lentiviral Vector, Stem Cell

\section{Background}

Systematically delivery of pharmacologic agents into central nervous system, not only often have transient efficacy and produce undesirable side effects but also blood barrier is a major limitation for it [1]. In recent years, gene therapy has been one of promising therapeutic procedures that has resolved some of challenges related to the treatment of neurological injuries and several pre-clinical and clinical attempts have been done in different neurological disorders [2]. The most significant problem for in vivo gene therapy of nervous system is the introduction of exogenous nucleic acid into the targeted cells. In neurodegenerative conditions the expression of cell receptors or other factors required for gene delivery into the cell may be affected by disorder that may lead to decrease the efficiency of gene delivery; also damaged cells will have high rate of death after genetically manipulation procedures [3,
4].

To overcome such problems, ex vivo gene therapy and grafting of genetically modified, may be preferable to in vivo gene therapy [5]. Ex vivo manipulation of cells can provide opportunity to control different biological characters such viability or immunogenicity of genetically engineered cells to select more appreciate vehicle cells tissue [6], as well as, to resolve problems related to unwanted infection of host cells. For these purposes, different primary or stem cells could be used as cellular vehicles for gene delivery [7]. For using in nervous system, selected cells should have characters such compatibility, differentiation potency into neural or glial cells or stability after transplantation which lead to prevention of any interruption of structure or function of host tissue [5]; moreover, transplanted cells should not stimulate immune system and also not be tumorogenic in host tissue.

To transfer exogenous gene into such cellular vehicles, 
different vectors can be desired. Although non-viral vectors are safer than viral vectors in the regard of immunogenicity, they cannot introduce exogenous gene into cells permanently and they are not proper candidates for longterm purposes [8]. Virus based vectors are potent vehicles for gene transduction and easily penetrate cell and nucleus membranes, leading to temporal or permanent gene incorporation in host genome depended on the kind of viral vector [9]. Vectors derived from LV(Lentivirus), are capable to integrate into both dividing and non-dividing cells that is one of the most important features of them for gene delivery purposes and some clinical trials using them are underway $[10,11]$. Although their ability to integrate packaged gene in host genome can lead to long-term expression of desired gene, and resolve problems related for multiple gene deliveries, their random insertion in host genome may lead to changes in cell structure [12].

Recent study in our lab showed that USSCs (Unrestricted somatic stem cells) are appreciate candidate to use as cellular vehicle for ex vivo gene therapy in nervous system [13]. These multipotent stem cells, isolated and characterized with Kogler et al. [14], have shown promising results to use in nervous system cell and gene therapy; they have neuronal differentiation capacity [15] and have been suggested to use as cellular vehicles for gene therapy with different groups [16].

\section{Objectives}

One of challenges regarding use of lentiviral vectors for gene manipulation purposes is their random insertion that can lead to changes in host cell; considering this subject, in current study we investigated the influence of lentiviral transduction on the neuronal related characters of USSCs as candidate to use as cellular vehicles for indicating the probable and unwanted changes related to transduction procedure.

\section{Materials and Methods}

\subsection{USSCs Isolation and Culture}

In this experimental study, USSCs were isolated from HUCB (human umbilical cord blood) and characterized as described previously [17]. Briefly, the mononuclear cell fraction of HUCB was separated by density centrifugation over a Ficoll-Hypaque gradient $(\mathrm{d}=1.077 \mathrm{~g} / \mathrm{mL}$, Pharmacia-Amersham, Piscataway, USA). The mononuclear cell suspension was plated in initiation medium containing low-glucose Dulbecco's Modified Eagle's Medium (DMEM), (Gibco, UK) supplemented with 30\% fetal calf serum (FCS) (Gibco, UK) , 10 - 7 M dexamethasone (SigmaAldrich, USA) and 1\% streptomycine-penicilin (Gibco, USA). After 15 - 20 days, USSC colonies were detached with $0.25 \%$ Trypsin-EDTA (Gibco, UK) and cultured in DMEM medium supplemented with 15\% fetal bovine serum (FBS) (Gibco, UK) and $1 \%$ streptomycine-peniciline in a humidified atmosphere of $95 \%$ air and $5 \% \mathrm{CO}_{2}$ at $37^{\circ} \mathrm{C}$. Isolated USSCs were characterized using flow cytometry and multilineage capacity for differentiation into cells of the three germ line layers.

\subsection{Construction of Transfer Vector and Virus Production}

The lentiviral vector containing GFP expression cassette was kindly gifted by Jalali et al. from Stony Brook university [13], USA and lentiviral packaging plasmids, psPAX2 and pMD2.G, were obtained from Addgene company. Virus production was done using transfection reagent lipofectamin 2000 (Invitrogen, UK). Briefly, $4 \times 10^{6} 293 \mathrm{~T}$ cells (Pasteur, Iran) were plated in $10 \mathrm{~cm}^{2}$ plate in $10 \mathrm{~mL}$ of the DMEM medium and transfected following day with $20 \mu \mathrm{g}$ transgene plasmid, $6 \mu \mathrm{g}$ pMD2G plasmid, $15 \mu \mathrm{g}$ psPAX2 plasmid and $800 \mu \mathrm{L}$ lipofectamin 2000 (Invitrogen, UK). Medium was removed around 6 - 7 hours post-transfection and $10 \mathrm{~mL}$ of fresh pre-heated medium was replaced. The virus contained supernatants were collected at 24, 36 and 48 hours post transfection and lentiviral stocks, filtered through $0.45 \mathrm{~mm}$ filters and concentrated by ultracentrifuge (Sigma 3-30 K, USA) in 40000 rpm for 2.5 hours. The titers of different lentiviral stocks generated in this study were determined by FACS and the percentage of green fluorescent cells was determined by Win MDI 2.8 software.

\subsection{Cell Transduction}

For cell infection, USSCs were plated in 24 well plates and 24 hours after plating, cell medium was harvested and $300 \mu \mathrm{L}$ medium containing adequate virus particles and $8 \mu \mathrm{g} / \mathrm{mL}$ polybrene (Sigma-Aldrich, USA) was replaced; 16 hours after cell transduction, supernant medium was collected and replaced by ordinary culture medium; the efficiency of transduction was measured after 72 hours on the basis of the percentage of GPF expressing cells under the fluorescent microscopy observation.

\subsection{Cell Viability Assay}

To assay the viability of transduced USSCs, the (3-(4, 5-dimethylthiazolyl-2)-2, 5-diphenyltetrazolium bromide) MTT assay was performed as described by ATCC protocol. Briefly, $10^{5} / \mathrm{mL}$ cells were plated in each well of 96 well and 24 hour after incubation $10 \mu$ L MTT reagent (Sigma, USA) was added to each well. Four hours after incubation in darkness, $100 \mu$ L DMSO (Dimethyl sulfoxide) (Sigma, USA) was added to each well and absorbance was red at $570 \mathrm{~nm}$. 


\subsection{Real-Time PCR}

Total RNA was extracted using Qiazol (Qiagen, USA) according manufactory's protocol and cDNA was synthesized from $2 \mu \mathrm{L}$ of RNA using Mu-MLV reverse transcriptase (Fermentas, Germany). Real-time PCR was performed using SYBR Green Premix Ex Taq II (TaKaRa, Bio Inc. Japan) on a Rotor Gene 6000 instrument (Corbett) and Rotor-gene Q software (Corbett) was used for data analysis. All reactions were run duplicate and housekeeping gene $\beta 2 \mathrm{M}$ was used for normalization of gene expression. Forward and reveres primers for analyzed genes have been listed in Table 1.

Table 1. List of Primers Used for Real Time PCR Reaction [Sense (Up) and Antisense (Down)]

\begin{aligned} & \hline \multicolumn{1}{c}{ Genes } Primers \\ & \hline Nestin \\ & \hline F: 5'GAAGGTGAAGGGCAAATCTG3' \\ & \hline R: 5'CCTCTTCTTCCCATATTTCCTG' \\ & \hline BDNF \\ & \hline F: 5'GTGAATTGATAATAAACTGTCCTC3' \\ & \hline R: 5'TAATTCCAACGCTATCAGAAG3' \\ & \hline FDNF 5'GAAATAGAAGGCTGGTGAGTG3' \\ & \hline R: 5'ACGACAGGTCATCAT CAA AG3' \\ & \hline NGF \\ & \hline F: 5'GGACCCAATAACAGTTTTACC3' \\ & \hline R: 5'GAACAACATGGACAT TACGC3' \\ & \hline F: \\ & \hline F: 5'GATCGGAGCCAAGTTCTG3' \\ & \hline\end{aligned}

\subsection{Neural Differentiation}

Neural differentiation of USSCs was performed as described previously [18]. Briefly, untransfected and transfected USSCs incubated with $50 \mathrm{ng} / \mathrm{mL} \mathrm{NGF,} 20 \mathrm{ng} / \mathrm{mL}$ bFGF, $1 \mathrm{mM}$ dibutyryl-cAMP, 0.5 mM IBMX (3-Isobutyl-methylxanthine) and 1- 7 M ATRA(all-trans retinoic acid), (all from Sigma-Aldrich, USA) up to 14 days and neuronal differentiation was verified according neural specific $\beta$-tubulin III, $\gamma$-enolase and MAP-2 expression.

\subsection{Immunocytochemistry Analysis}

Cells were fixed with $4 \%$ paraformaldehyde for $30 \mathrm{~min}$ utes, permeabilized with Triton X-100 (0.4\%) for $10 \mathrm{~min}$ utes, blocked with $5 \%$ goat serum and processed for immunocytochemistry using mouse anti-human $\beta$-tubulin III (Santa-Cruz, 1:200, USA), mouse anti-human $\gamma$-enolase (Santa Cruz; 1:300, USA), mouse anti-human MAP2 (Abcam, 1:500, USA) antibodies. Donkey anti mouse IgGPE (eBioscience, 1:400, USA) was used as secondary antibody and cell nuclei were labeled with 4,6-diamidino-2phenylindole (DAPI) (Sigma-Aldrich, USA).

One-way ANOVA test was performed for statically analysis of data. Values were expressed as the mean \pm SEM and differences were considered significant at $\mathrm{P}<0.05$. For statistical comparison of real time PCR results, REST software (2009, Qiagen) was used.

\section{Results}

\subsection{Transductin of USSCs With GFP-Lentiviral Vector}

Human USSCs were transduced with lentivirus derived vector containing GFP gene under the control of EF $\alpha$ promoter for stable integration of exogenous plasmid in their genome and GFP served as control by marking all cells that received the target plasmid. Observation of GFP expressing cells under the fluorescent microscopy showed almost $100 \%$ of USSCs were infected successfully when exposed to a multiplicity of infection (MOI) of 10 of produced viruses (Figure 1).

\subsection{Effect of Lentiviral Transduction on the Viability of USSCS}

Followed by lentiviral transduction, MTT analysis was performed to investigate the effect of virus infection and gene transduction on the viability and proliferation of USSCs. Obtained data indicated that $94 \%$ of transduced cells were viable after lentiviral transduction and no significant adverse effect on cell viability and proliferation observed when compared to non-transduced control cells at $\mathrm{P}<0.05$ (Figure 2).

\subsection{Effect of Lentiviral Transduction on the Neuronal Differenti- ation of USSCS}

Native and lentiviral transduced USSCs were induced toward neuronal lineage differentiation in the presence of inducing factors to compare their abilities and to investigate the effect of viral transduction on the differentiation potency of USSCs. Following 14 days neuronal induction, results showed both groups started to get neural-like phenotypes with condensed nucleus and thin and long dendrite or axonal like branches. Immunostaining against 

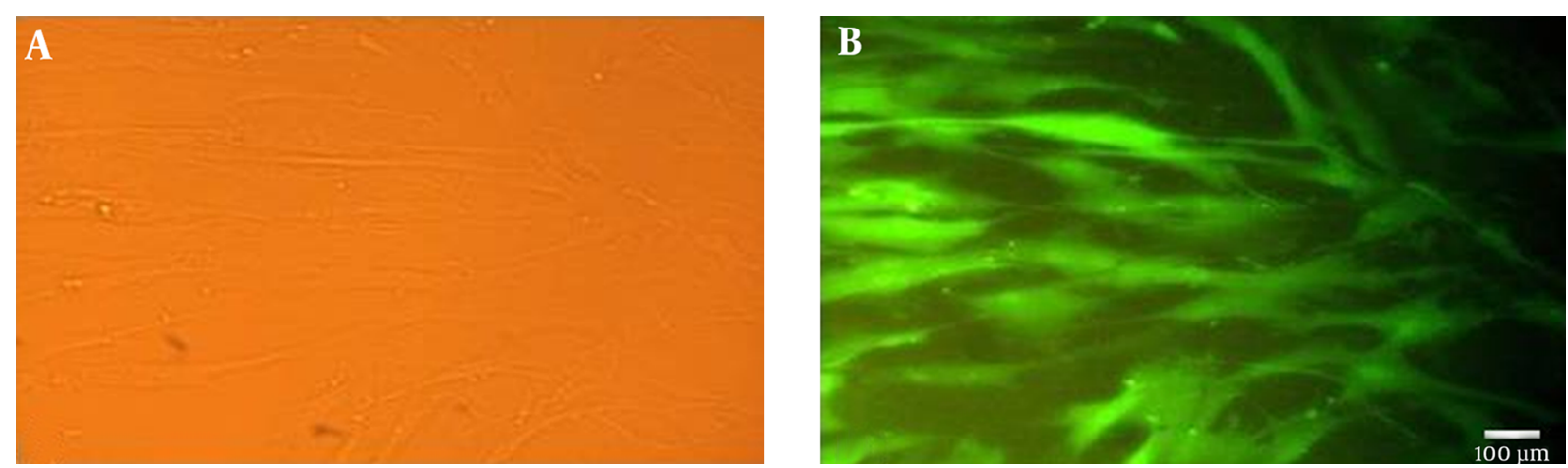

Efficiency of transduction was confirmed by visualizing GFP expression. A, light microscope; B, invert fluorescent microscope. Scale bar is $100 \mu \mathrm{m}$.

Figure 2. The MTT Assay Showing the Viability Percentage of Lentivirus Transduced Unrestricted Somatic Stem Cells

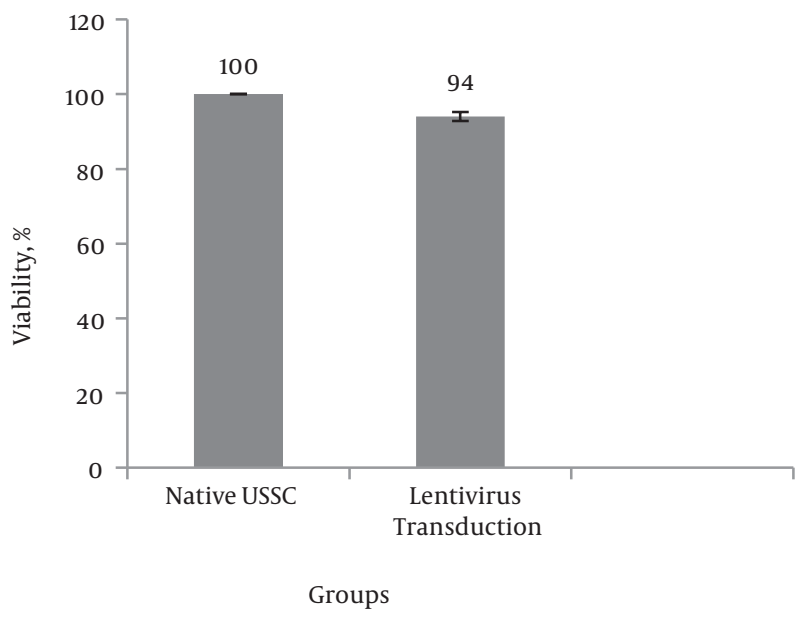

There was no significant difference between native USSCs as control andtransduced groups at $\mathrm{P}<0.05$. Values are represented as Mean \pm SEM.

neuron specific $\beta$-tubulin III and $\gamma$-enolase showed significant expression of these markers in both groups of differentiated cells but no expression of MAP-2 marker neither in transduced nor in untransduced USSCs induced toward neural differentiation (Figure 3).

4.4. Effect of Lentiviral Transduction on the Expression of Neuronal Genes

The expression of the Nestin, as an early neuronal gene, $\gamma$-enolase and $\beta$-tubulin III, as late neuronal genes, and also NGF, GDNF and BDNF, as neurotrophic factors, were compared before and after viral transduction of USSCs using real time PCR. Comparison of CT values indicated that ex- pression of these genes changed after transduction from 1.42 times in NGF to 4.36 times in $\gamma$-enolase (Figure 4 ).

\section{Discussion}

In this study we investigated the effect of GFP containing LV transduction on the viability, differentiation potency and neural gene expression of USSCs. Obtained results showed transduction of LV did not have cytotoxic effects on USSCs and transduced USSCs had high rate of viability and they proliferated and expanded like native USSCs; this result could be helpful to provide sufficient manipulated cells for transplantation purposes [19].

In the presence of neuronal inducing factors, lentiviral transduced USSCs differentiated into neural like cells comparable to untransduced USSCs and differentiated cells expressed neuronal specific $\beta$-tubulin III and $\gamma$-enolase proteins. These results indicated that lentivirus transduction did not affect neural differentiation potency of USSCs and preserved them in multilineage situation; these indicated that engineered USSCs could be used as cellular vehicle and can be transplanted to host tissue after neuronal differentiation that leads to higher compatibility of these cells with nervous system and may help to efficient regeneration of damaged tissue [5].

Analysis of lentiviral transduction on the gene expression profile of transduced USSCs showed the expression of early and late neural genes and also some neurotrophic factors related genes, changed few in comparison with untransduced USSC and lentiviral transduction did not changed the expression of studied genes so strongly and kept their expression comparable to innate condition.

The most notable advantage of ex vivo gene therapy may be the possibility for special controls before transplantation of modified cells and also prevention of un- 
Figure 3. Immunohistochemical Analysis of Neural Differentiation of Lentiviral Transduced USSCs in Presence of Inducing Factors
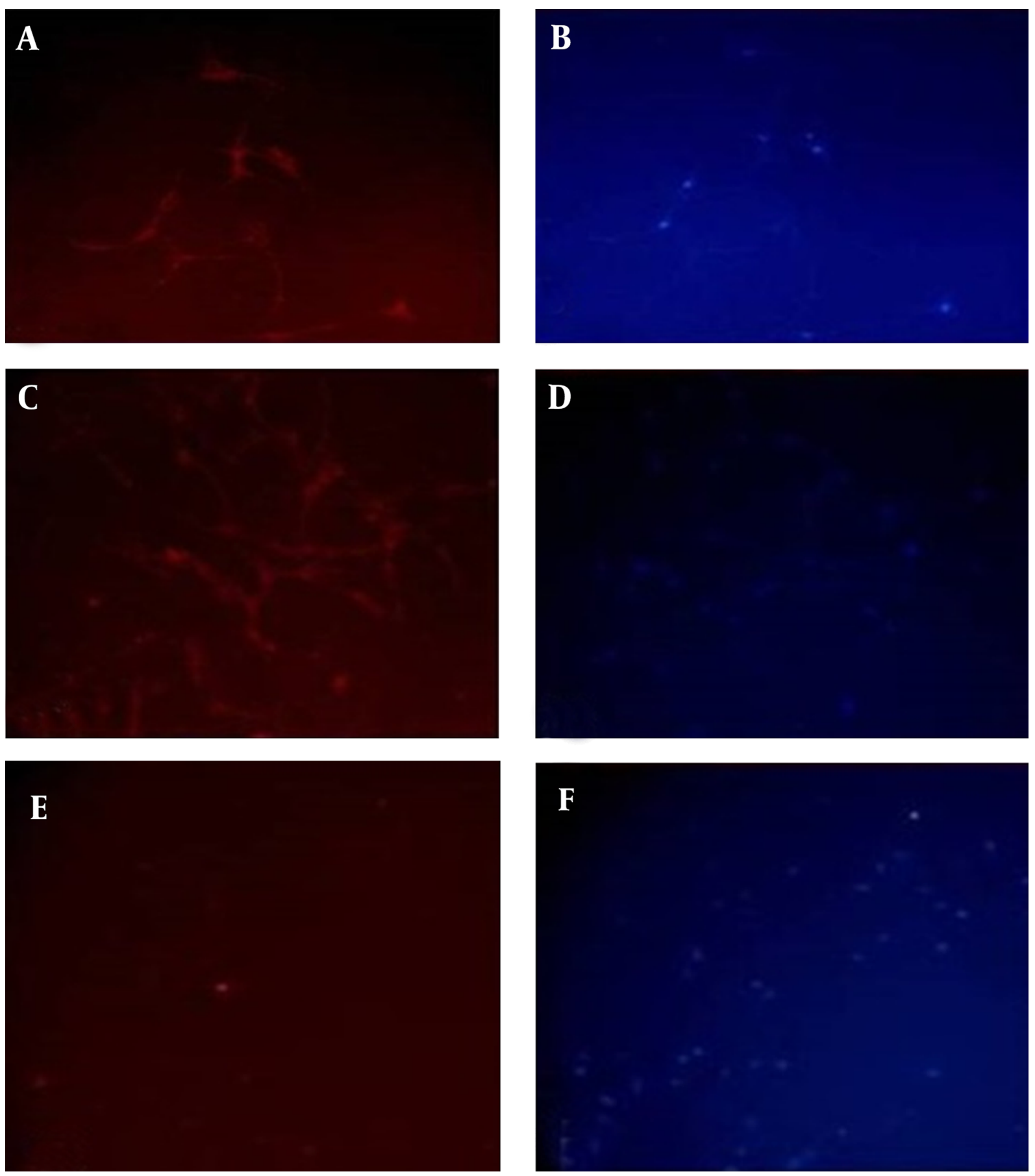

Visualizing under invert fluorescent microscope; A and B, $\beta$-tubulin III marker and DAPI; C and D, $\gamma$-enolase marker and DAPI; E and F, MAP-2 and DAPI. Scale bar is $100 \mu \mathrm{m}$.

desired side effects of direct delivery of vector especially in the case of viral vectors [20]. Beside to that engineered stem cells can transfer exogenous gene and can restore the expression of damaged factor, such cells can be a rich source of different growth factors or cytokines providing trophic support of the injured nervous system 
Figure 4. Comparison of Neural Related Genes Expression in Lentiviral Transduced USSCs vs. Untransduced USSCs Using Real Time PCR

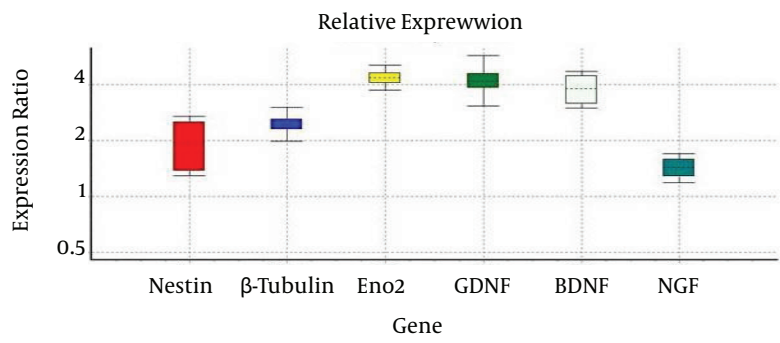

Integration of lentiviral vector into the genome of USSCs led to slight increase in expression of investigated genes. Analysis with REST 2009 software (Qiagen).

[6]. There is evidence suggesting that human neural stem cells, human umbilical cord blood stem cells and murine mesenchymal stem cells secrete GDNF and BDNF (glial cell-and brain-derived neurotrophic factors), IGF-1 (insulin like growth factor) and VEGF (vascular endothelial growth factor), which may protect dysfunctional motor neurons and prolongs the lifespan animal models of neurodegenerative diseases [21]. Primary astrocytes were genetically modified ex vivo to express recombinant GDNF (glial cell line-derived neurotrophic factor) and subsequently were tested for their ability to provide neuroprotection to dopaminergic neurons in a 6-OHDA (6-hydroxydopamine) mouse model of Parkinson's disease which indicated that continuous exposure to GDNF provided by transgenic astrocytes caused marked protection of nigral dopaminergic neurons [22].

Unrestricted somatic stem cells as a subtype of UCBSCs show appreciate properties for using in neurodegenerative disorders; they produce vast variety of cytokines and they are able to differentiate to neuronal cells [23, 24]. Transplantation of human USSCs into the rat model of acute spinal cord injury reduced the lesion size and promoted the regeneration of damaged axons [25]. Grafted USSCs showed the high tropism into the lesion sites, especially apoptotic neurons, which was related to expression of c-MET receptors and interaction with HGF secreted by apoptotic neurons [26]. In vitro and in vivo models showed that USSCs when injected into uninjured site strongly migrated toward lesion center and did not attract by uninjured site [25]; this character of USSCs can help the targeted transfer of gene toward the site of damage, especially toward apoptotic neurons, and makes them appreciate biological vehicles for gene transferring. For genetically modification of USSCs and creation of exogenous gene containing USSCs, different kind of vectors can be used, however, for stable transduction, vector should be able to incorporate exogenous gene in genome of these cells and lead to constant gene transfer [27]. Retroviral and lentiviral vectors both integrate into host genome permanently but because of ability to transduce non-dividing cells, lentiviral vectors have special advantage in compare to retroviral vectors and they are able to carry close to $10 \mathrm{~kb}$ of exogenous gene [28].

Ideally for gene therapy purposes vector should integrate to host genome without disturbing host cell genomic profile [9]. In the case of LV, random integration of them may lead to some changes in gene expressions and it is critical to check such changes before using modified cells [29].

In summary, results from current study showed that neural related properties of lentiviral transduced USSCs were comparable with native USSCs regarding the survival, neural differentiation potency and the expression of neuronal related genes and engineered USSCs did not undergo significant changes during the transduction procedure. On the basis of these results, we suggest that lentiviral vectors could be suitable vectors to transfer desirable therapeutic gene into USSCs to create one cellular vehicle for using in nervous system ex vivo gene therapy.

\section{Acknowledgments}

This research is concerning $\mathrm{PhD}$ thesis (IranDoc: 2114042) in department of cell and developmental biology of Kharazmi university and department of molecular biology and genetic engineering of stem cell technology research center, Tehran, Iran.

\section{Footnotes}

Authors' Contribution: All authors had equal role in design, work, statistical analysis and manuscript writing.

Funding/Support: Molecular biology and genetic engineering group of stem cell technology research center, Tehran.

\section{References}

1. Pardridge WM. Molecular Trojan horses for blood-brain barrier drug delivery. Curr Opin Pharmacol. 2006;6(5):494-500. doi: 10.1016/j.coph.2006.06.001. [PubMed:16839816].

2. Lim ST, Airavaara M, Harvey BK. Viral vectors for neurotrophic factor delivery: a gene therapy approach for neurodegenerative diseases of the CNS. Pharmacol Res. 2010;61(1):14-26. doi: 10.1016/j.phrs.2009.10.002. [PubMed: 19840853].

3. Jakobsson J, Ericson C, Rosenqvist N, Lundberg C. Lentiviral vectors. Int Rev Neurobiol. 2003;55:111-22. [PubMed: 12968533].

4. Manfredsson FP, Mandel RJ. The development of flexible lentiviral vectors for gene transfer in the CNS. Exp Neurol. 2011;229(2):201-6. doi: 10.1016/j.expneurol.2011.03.013. [PubMed: 21459087]. 
5. Snyder EY, Senut MC. The use of nonneuronal cells for gene delivery. Neurobiol Dis. 1997;4(2):69-102. doi:10.1006/nbdi.1997.0138. [PubMed: 9331899].

6. Hwang DH, Jeong SR, Kim BG. Gene transfer mediated by stem cell grafts to treat CNS injury. Expert Opin Biol Ther. 2011;11(12):1599-610. doi: 10.1517/14712598.2011.631908. [PubMed: 22017608].

7. Lindvall $\mathrm{O}$, Kokaia $\mathrm{Z}$. Stem cells in human neurodegenerative disorders-time for clinical translation?. J Clin Invest. 2010;120(1):2940. doi: 10.1172/JCI40543. [PubMed: 20051634].

8. Roybal JL, Santore MT, Flake AW. Stem cell and genetic therapies for the fetus. Semin Fetal Neonatal Med. 2010;15(1):46-51. doi: 10.1016/j.siny.2009.05.005. [PubMed:19540822].

9. Hermens WT, Verhaagen J. Viral vectors, tools for gene transfer in the nervous system. Prog Neurobiol. 1998;55(4):399-432. [PubMed: 9654386].

10. Snyder BR, Boulis NM, Federici T. Viral vector-mediated gene transfer for CNS disease. Expert Opin Biol Ther. 2010;10(3):381-94. doi: 10.1517/14712590903514074. [PubMed: 20132059].

11. Azzouz M, Kingsman SM, Mazarakis ND. Lentiviral vectors for treating and modeling human CNS disorders. J Gene Med. 2004;6(9):951-62. doi: 10.1002/jgm.600. [PubMed:15352068].

12. Michel G, Yu Y, Chang T, Yee JK. Site-specific gene insertion mediated by a Cre-loxP-carrying lentiviral vector. Mol Ther. 2010;18(10):1814-21. doi: 10.1038/mt.2010.150. [PubMed: 20628360].

13. Jalali H, Parivar K, Nabiuni M, Soleimani M. Unrestricted somatic stem cells as vehicle for nerve growth factor gene transfer. Neurol Res. 2013;35(6):553-60. doi: 10.1179/1743132813Y.0000000176. [PubMed: 23561736].

14. Kogler G, Sensken S, Airey JA, Trapp T, Muschen M, Feldhahn N, et al. A new human somatic stem cell from placental cord blood with intrinsic pluripotent differentiation potential. J Exp Med. 2004;200(2):12335. doi: 10.1084/jem.20040440. [PubMed: 15263023]

15. Fallahi-Sichani M, Soleimani M, Najafi SM, Kiani J, Arefian E, Atashi A In vitro differentiation of cord blood unrestricted somatic stem cells expressing dopamine-associated genes into neuron-like cells. Cell Biol Int. 2007;31(3):299-303. doi: 10.1016/j.cellbi.2006.11.011. [PubMed: 17196845].

16. Rosenkranz K, Meier C. Umbilical cord blood cell transplantation after brain ischemia-from recovery of function to cellular mechanisms. Ann Anat. 2011;193(4):371-9. doi: 10.1016/j.aanat.2011.03.005. [PubMed: 21514122].

17. Hafizi M, Bakhshandeh B, Soleimani M, Atashi A. Exploring the enkephalinergic differentiation potential in adult stem cells for cell therapy and drug screening implications. In Vitro Cell Dev Biol Anim. 2012;48(9):562-9. doi: 10.1007/s11626-012-9546-4. [PubMed: 23054438]

18. Iwaniuk KM, Schira J, Weinhold S, Jung M, Adjaye J, Muller HW, et al. Network-like impact of MicroRNAs on neuronal lineage differentia- tion of unrestricted somatic stem cells from human cord blood. Stem Cells Dev. 2011;20(8):1383-94. doi: 10.1089/scd.2010.0341. [PubMed: 21067317].

19. Ali H, Bahbahani H. Umbilical cord blood stem cells - potential therapeutic tool for neural injuries and disorders. Acta Neurobiol Exp (Wars). 2010;70(3):316-24. [PubMed: 20871652].

20. Gage FH, Fisher LJ, Jinnah HA, Rosenberg MB, Tuszynski MH, Friedmann T. Grafting genetically modified cells to the brain: conceptual and technical issues. Prog Brain Res. 1990;82:1-10. [PubMed:1981271].

21. Vishwakarma SK, Bardia A, Tiwari SK, Paspala SA, Khan AA. Current concept in neural regeneration research: NSCs isolation, characterization and transplantation in various neurodegenerative diseases and stroke: A review. J Adv Res. 2014;5(3):277-94. doi: 10.1016/j.jare.2013.04.005. [PubMed: 25685495].

22. Cunningham LA, Su C. Astrocyte delivery of glial cell line-derived neurotrophic factor in a mouse model of Parkinson's disease. Exp Neurol. 2002;174(2):230-42. doi: 10.1006/exnr.2002.7877. [PubMed:11922664].

23. Kogler G, Radke TF, Lefort A, Sensken S, Fischer J, Sorg RV, et al. Cytokine production and hematopoiesis supporting activity of cord blood-derived unrestricted somatic stem cells. Exp Hematol. 2005;33(5):573-83. doi: 10.1016/j.exphem.2005.01.012. [PubMed: 15850835].

24. Trompeter HI, Abbad H, Iwaniuk KM, Hafner M, Renwick N, Tuschl T, et al. MicroRNAs MiR-17, MiR-20a, and MiR-106b act in concert to modulate E2F activity on cell cycle arrest during neuronal lineage differentiation of USSC. PLoS One. 2011;6(1):e16138. doi: 10.1371/journal.pone.0016138. [PubMed: 21283765].

25. Schira J, Gasis M, Estrada V, Hendricks M, Schmitz C, Trapp T, et al. Significant clinical, neuropathological and behavioural recovery from acute spinal cord trauma by transplantation of a well-defined somatic stem cell from human umbilical cord blood. Brain. 2012;135(Pt 2):431-46. doi: 10.1093/brain/awr222. [PubMed: 21903726].

26. Trapp T, Kogler G, El-Khattouti A, Sorg RV, Besselmann M, Focking M, et al. Hepatocyte growth factor/c-MET axis-mediated tropism of cord blood-derived unrestricted somatic stem cells for neuronal injury. J Biol Chem. 2008;283(47):32244-53. doi: 10.1074/jbc.M800862200. [PubMed: 18628208].

27. Washbourne P, McAllister AK. Techniques for gene transfer into neurons. Curr Opin Neurobiol. 2002;12(5):566-73. [PubMed: 12367637].

28. Trono D. Lentiviral vectors: turning a deadly foe into a therapeutic agent. Gene Ther. 2000;7(1):20-3. doi: 10.1038/sj.gt.3301105. [PubMed: 10680011].

29. Staunstrup NH, Moldt B, Mates L, Villesen P, Jakobsen M, Ivics Z, et al. Hybrid lentivirus-transposon vectors with a random integration profile in human cells. Mol Ther. 2009;17(7):1205-14. doi: 10.1038/mt.2009.10. [PubMed:19240688]. 